\title{
Salivary Alpha-amylase Enzyme and Salivary Cortisol Level in Depression after Treatment with Fluoxetine
}

\author{
Andi Jayalangkara Tanra D, Hawaidah Madeali, Mayamariska Sanusi*, Saidah Syamsuddin, Sonny Teddy Lisal \\ Department of Psychiatry, Faculty of Medicine, Hasanuddin University, Makassar, Indonesia
}

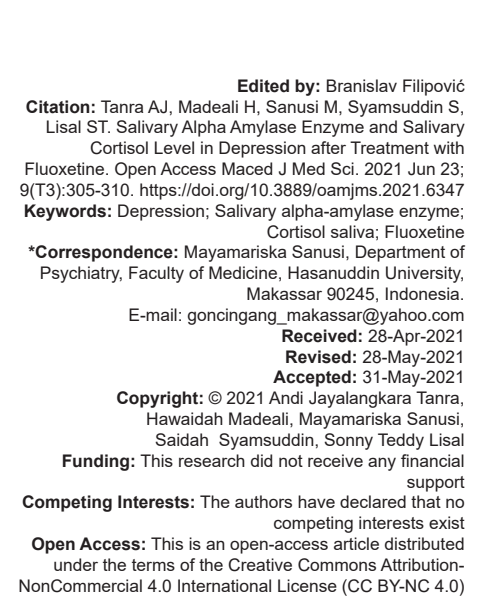

\begin{abstract}
BACKGROUND: Hypothalamic-pituitary-adrenal axis and its end product cortisol have been extensively investigated in patients with depressive disorders for many years. Recently, salivary alpha-amylase (sAA) had emerged as a new biomarker with non-invasive and more convenience protocol for measuring sympathetic activity which was also associated with depression. Selective Serotonin Reuptake Inhibitor is antidepressant drug extensively used to treat depression.

AIM: The aim of this study was to determine whether a decrease of sAA and salivary cortisol levels could be observed in subjects with depression who were treated by fluoxetine.

METHODS: The total subjects were 25 depressed subjects and 10 healthy controls. sAA was examined before therapy and after 2, 4, and 6 weeks of fluoxetine administration using a portable colorimeter. Salivary cortisol was examined before therapy, after 4 and 6 weeks of fluoxetine administration with the Elisa method. The therapeutic effect was assessed with Hamilton Depression Rating Scale (HDRS).RESULTS: sAA and cortisol level were significantly decreased after fluoxetine administration $(p<0.001)$ followed by at least $50 \%$ reduction of HDRS scores after 6 weeks of fluoxetine administration. Levels of SAA and cortisol were higher in the depression group than in the healthy control.
\end{abstract}

CONCLUSIONS: Measurement of SAA levels can be used as a potential biomarker of therapeutic response in depressed patients in addition to salivary cortisol.

\section{Introduction}

Major depressive disorder (MDD) is the leading cause of disability worldwide and is a major contributor to the overall global burden of disease [1]. In Indonesia, the prevalence of mental disorders indicated by symptoms of depression and anxiety increased from $6 \%$ in 2013 to $6.1 \%$ in 2018 [2]. The high level of stress was thought responsible for the increased rate of mental disorder. Stress is defined as a state of threatened homeostasis following exposure to extrinsic or intrinsic adverse forces. Acute stress refers to stress that occurs in minutes or hours, whereas chronic stress persists for days, weeks, or months. Stress was associated with depression and could precipitate other depressive episodes, including severity, duration, and the natural course of the disorder [3], [4]. In response to a stressor, two major biological stress systems are activated: the Sympathetic Nervous System (SNS) and the Hypothalamic-Pituitary-Adrenal (HPA) axis. Across life, the repetition or chronic activation of these systems could lead to changes in functioning, impaired or inadequate responses to subsequent challenges [5], [6].

At present, the diagnosis of MDD mainly relies on clinical examination and subjective evaluation of depressive symptoms. At present, there is no specific biomarker for MDD have been identified to date. However, biomarker can be helpful in the treatment choice and in predicting the course of the disorder during the early stages [7], [8].

Cortisol and alpha-amylase are both markers of the human stress system but have a different mechanism. Over the past years, the functioning of the HPA axis and its end-product cortisol had received much attention. Salivary cortisol, a commonly used biomarker of the stress response, has been used in a range of clinical study of stress-related diseases, including depression [9], [10]. In a meta-analysis, Stetler and Miller summarized four decades of research into HPA axis activation and depression and concluded that there was a tendency for an increase of HPA axis activation in depressed subjects. They also noted that results varied considerably across studies and that several studies showed decreased instead of increased activation of the HPA axis [11]. Salivary alpha-amylase (sAA) is an enzyme that hydrolyses starch in the oral cavity; secreted by parotid gland under autonomic regulation and highly sensitive to stress-related changes. SAA has emerged as a new biomarker for responses to psychosocial stress within the SNS. Activation of the SNS results in norepinephrine (NE) release that may subsequently 
elicit the release of SAA by the salivary glands. NE is involved intrinsically with the stress response system, and chronic stress in depression would trigger the release of NE from SNS. Few studies that examined sAA level in relation to depressive symptoms show a tendency towards increased SAA level in depressed versus non-depressed group [10], [11], [12].

Ishitobi et al. (2010) compared basal level of salivary cortisol and SAA in unremitted and remitted MDD patients as well as in healthy controls. It was found that sAA and cortisol levels in unremitted patients were significantly elevated compared to controls and remitted patients. It suggested that $\mathrm{SAA}$ may be a state-dependent marker of MDD in addition to salivary cortisol. Bauduin et al. suggest that sAA at awakening may be a valuable candidate biomarker specifically for MDD [13], [14].

Selective Serotonin Reuptake Inhibitors (SSRIs) are antidepressant drugs extensively used in MDD. Interestingly, besides increasing serotonergic neurotransmission, SSRIs might also alter HPA-axis disturbances [15]. In MDD, the SSRI fluoxetine decreased corticotropin-releasing hormone [16]. Effects of antidepressants, including SSRIs on the HPA-axis, occurred mainly in MDD-patients who are responsive to treatment. Therefore, it has been suggested that resolving HPA-axis abnormalities during MDD treatment indicates SSRI response [17], [18].

A study by Piwowarska et al. concluded that patients who were successfully treated with fluoxetine showed a significant decrease in Hamilton Depression Rating Scale (HDRS) scores and decreased cortisol secretion after 6 weeks of treatment [19]. However, after 5 weeks of treatment with SSRI or the nonSSRI, HPA axis activities only decrease in non-SSRI responders [17]. Another study showed that the administration of SSRIs did not show any changes in sAA levels [20].

Evaluation of cortisol levels is mostly done for research purposes, it is still rarely used in daily clinical practice considering that not all health facilities can carry out cortisol measurements and the costs needed are relatively more expensive. The measurement of sAA level using optical detection method through saliva was introduced as a non-invasive and more convenience protocol. It does not make the subjects to be stress as it gives ease in taking samples; besides, time needed is relatively short, easy to do anywhere and anytime without being reliant on the assistance of laboratory staff.

In a previous study at our department shown that sAA level in subject with depression was significantly higher than the normal population. In addition, an increase in HDRS scores in patients with depression was followed by a significant increase in the levels of the sAA enzyme [21]. In this study, we compared the level of $\mathrm{SAA}$ and salivary cortisol in the depressed subject before and after treatment with fluoxetine. We hypothesized that sAA enzyme and salivary cortisol would be lower after treatment with fluoxetine.

\section{Methods}

\section{Patient sample}

The study included 25 subjects with MDD either inpatient or outpatient, who were admitted for the first time to Psychiatric clinic at Wahidin Sudirohusodo Hospital, Makassar, Indonesia and its network. The MDD diagnosed based on criteria of the Diagnostic and Statistical Manual of mental Disorder, $5^{\text {th }}$ edition (DSM-V). The ages of subjects were 18-45 years old. Exclusion criteria were comprised of the following: chronic metabolic and oral disease, required adrenergic agonist or antagonist drugs, required corticosteroid therapy and comorbidity with other psychiatric disorders. The healthy controls (10 subjects) were a civil servant in health service center, 18-45 years old with no history of mental disorder and met the same exclusion criteria with depressed subjects. All subjects were provided with complete written and oral descriptions of the study, written informed consent was also obtained. The protocol was approved by the local ethics committee.

\section{Procedures}

To control for variations of sAA and cortisol levels, salivary collection was performed between 09:00 AM and 14:00 PM. We measured saliva samples four times, before treatment, 2, 4, and 6 weeks after treatment. sAA activity was measured using the cocorometer (Nipro Corp., Japan) according to the manufacturer's protocol. Saliva was sampled by directly immersing a salivasampling strip in saliva under the tongue for $30 \mathrm{~s}$, and then the test strip was inserted into the monitor which revealed the nominal of sAA enzyme levels. It took only $1 \mathrm{~min}$ to measure the sAA enzyme level. The measurement of salivary alpha-amylase enzyme by this tool using optical detection method. The substrate (saliva) was collected using strip which contains a strip reagent so that if mixed: Gal-G2 (Galaktospyranosylmatosa) as the substrate will bind to chloro-nitrophenyl (CNP) chromogen compound to become Gal-G2-CNP (2-Chloro-4-nitrophenyl-4O-ßD-galactospyranosylmaltoside). This 2-Chloro4Nitrophenyl (CNP) reagent strip will hydrolyze the substrate to detect the sAA enzyme by producing a yellow product. Changes in the intensity of the color were in accordance with changes in the activity of the salivary alpha-amylase enzyme; the higher the intensity of the yellow color meant the higher the sAA enzyme levels. Then, the reagent strip was inserted into the optical analyzer, in which the device was capable of measuring the activity of sAA enzyme. 
The concentration of salivary cortisol was measured using DBC Cortisol kit and analyzed by ELISA assay. The absorbance measured on a microtiter plate reader. Saliva samples were collected immediately using the passive drooling technique. The tubes were placed in a refrigerator at $-80^{\circ} \mathrm{C}$ and then thawed for analysis. Salivary cortisol was measured three times, before treatment, 4 , and 6 weeks after treatment. Subjects were refrained from eating, drinking, smoking, teeth brushing at least 30 min before sampling to minimize the effect of food or drink on the activity of the sAA and cortisol level, rinsing with mineral water, and expected to rest for about 10 min before sampling.

We assessed subjects with the HDRS for psychological status and efficacy of antidepressant therapy before treatment, 2, 4, and 6 weeks after treatment. Subjects were given $20-40 \mathrm{mg}$ of fluoxetine and benzodiazepine (alprazolam and lorazepam in equivalent dose for sedation). Control group was examined for SAA and salivary cortisol only one time, measurement results as a standard level for sAA and cortisol value.

The data were analyzed using IBM SPSS statistics for windows version 22 and presented as tables and graphs. Chi-square was used to assess the distribution of characteristics according to the sample group, Mann-Whitney was used to assess differences in initial cortisol levels and initial sAA between depressed and control groups. Wilcoxon signed-rank was used to assess differences in enzyme levels and HDRS scores between pre- and post-therapy. The test results are statistically significant if the value is $p<0.05$.

\section{Results}

Thirty-five subjects participated in the study. The subjects were divided in two groups, namely depressed group and control group. Ages of the subjects range between 22 and 45 years old. The mean age of subjects was 33.5 years $(S D=7.5)$. There were no significant differences in age, sex, and education level $(p>0.05)$. There was a difference in employment status where all the healthy control are an employee $(p=0.002)$.

Based on the collected data, SAA 0 (sAA initial) and cortisol_0 (cortisol initial) were taken before treatment. Significant initial sAA levels were observed higher in depressed group (52.0 kU/L) compared to control group (13.4 kU/L) with $p<0.001$. Initial cortisol levels were observed higher in depressed group $(24.1 \mathrm{ng} / \mathrm{dl})$ compared to control group $(7.9 \mathrm{ng} / \mathrm{dl})$ with $p<0.001$ shown in Table 1. Comparison of the sAA levels in every measurement compared with healthy control were shown in Figure 1. While comparison of salivary cortisol levels in every measurement compared with healthy control were shown in Figure 2.
Table 1: Comparison of initial SAA level and salivary cortisol level with healthy control

\begin{tabular}{llllll}
\hline Variable & Group & $\mathrm{n}$ & Mean & SD & $\mathrm{p}$ \\
\hline SAA 0 & Depression & 25 & 52.0 & 16.8 & 0.000 \\
& Control & 10 & 13.4 & 6.2 & \\
CORTISOL 0 & Depression & 25 & 24.1 & 14.5 & 0.000 \\
& Control & 10 & 7.9 & 3.2 & \\
\hline \multirow{2}{*}{ sAA: Salivary alpha-amylase. } & & & &
\end{tabular}

Comparison of the sAA levels in every measurement was shown in Figure 3. The sAA levels were found to be significantly decreased consistently after 2 weeks (50.28) kU/L, 4 weeks (46.0) kU/L, and 6 weeks (37.96) $\mathrm{kU} / \mathrm{L}$ of treatment. There was a significant reduction in SAA level after fluoxetine administration with $p<0.001$.

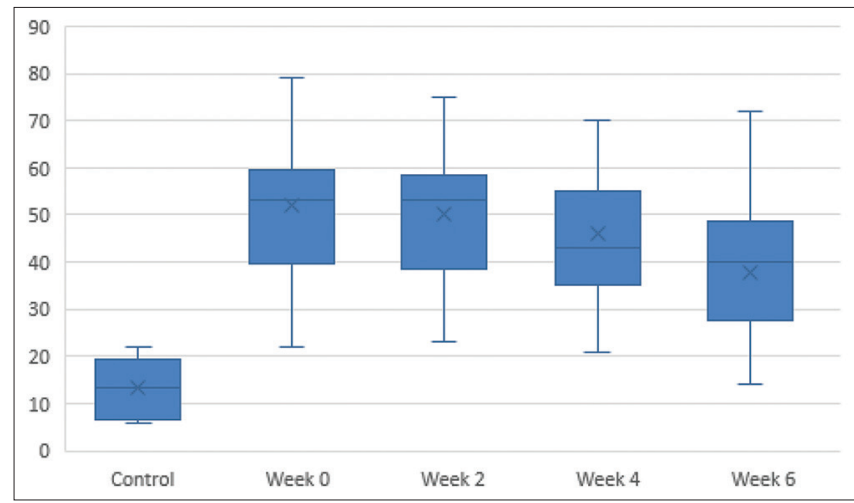

Figure 1: Comparison of salivary alpha-amylase level with healthy control

Comparison of salivary cortisol levels in every measurement was shown in Figure 4. The level of salivary cortisol was found to be significantly decreased consistently after 4 weeks $(19.32 .0) \mathrm{ng} / \mathrm{dL}$ and after 6 weeks (14.5) ng/dL of treatment. There was a significant reduction in salivary cortisol level after fluoxetine administration with $p<0.001$.

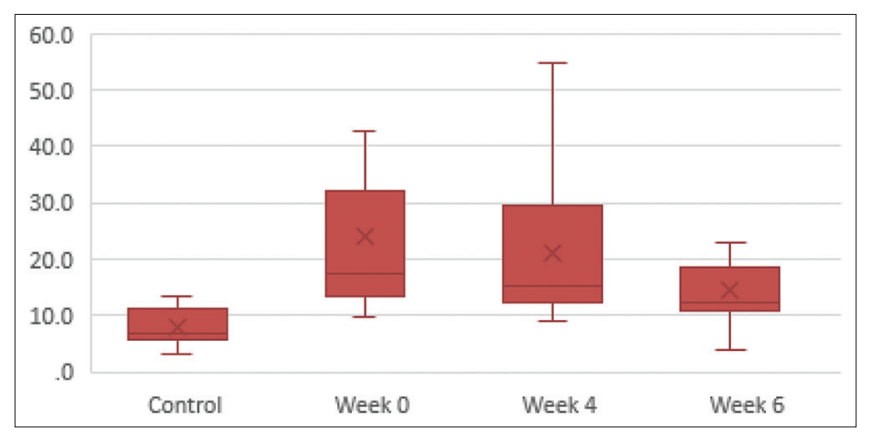

Figure 2: Comparison of salivary cortisol level with healthy control

The therapeutic effect was assessed with analysis of the percentage of the reduction of HDRS score compared to the initial values. HDRS scores were found to be significantly decreased consistently after 2 weeks (19.08), 4 weeks (15.6), and 6 weeks (12.12) of treatment. There is a significant reduction in HDRS score after fluoxetine administration with $p<0.001$. Six weeks were needed for the reduction by at least $50 \%(52.36 \%)$. Comparison of HDRS score in every measurement was shown in Figure 5. 


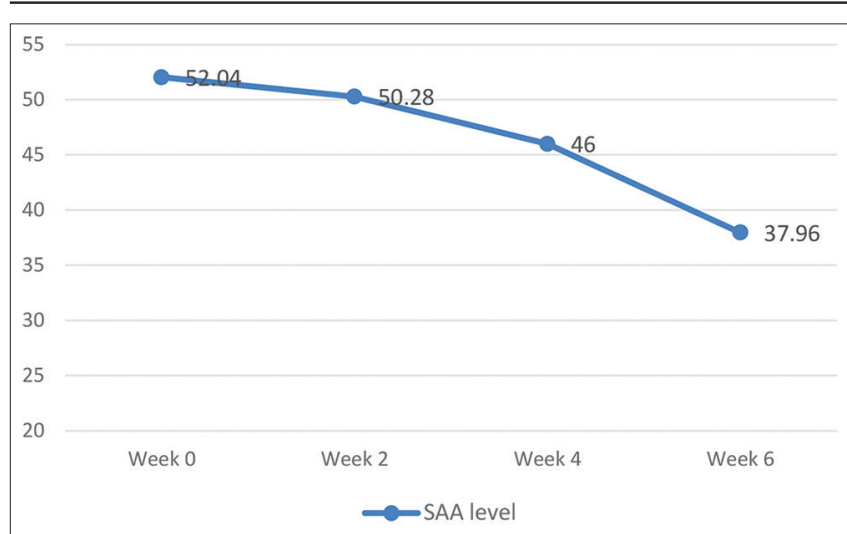

Figure 3: Comparison of salivary alpha-amylase level week 0, 2, 4, and 6

\section{Discussion}

This study was conducted to determine if there were differences in the SAA enzyme levels and the cortisol levels in depressed subjects before and after fluoxetine administration. We found that SAA levels were significantly higher in depressed subjects (52.04 \pm $16.84)$ compared to healthy controls $(13.40 \pm 6.24)$. The

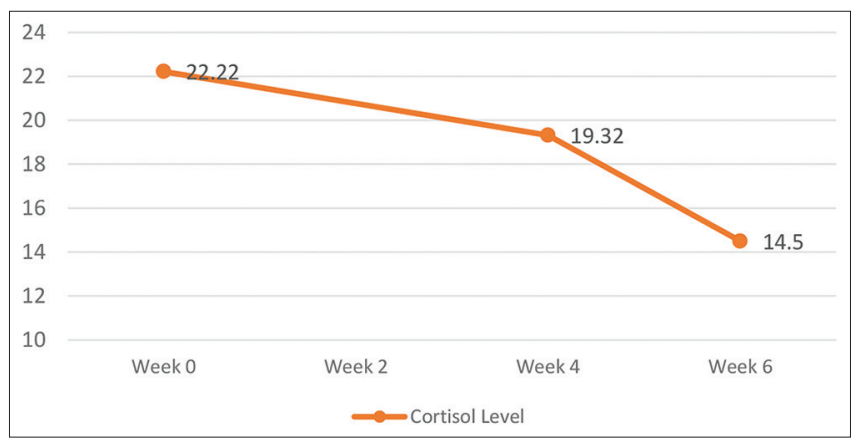

Figure 4: Comparison of cortisol level week 0 , 4, and 6

mean value of the initial sAA level in depressed subject is slightly higher than the previous research in our department which showed the average value $35.17 \pm$ $11.92 \mathrm{KU} / \mathrm{L}$ but lower than the average value in anxiety group that is $63.57 \pm 32.21$ [21]. It was suggested that $\mathrm{SAA}$ could be used as a marker for measuring sympathetic activity which associated with depression.

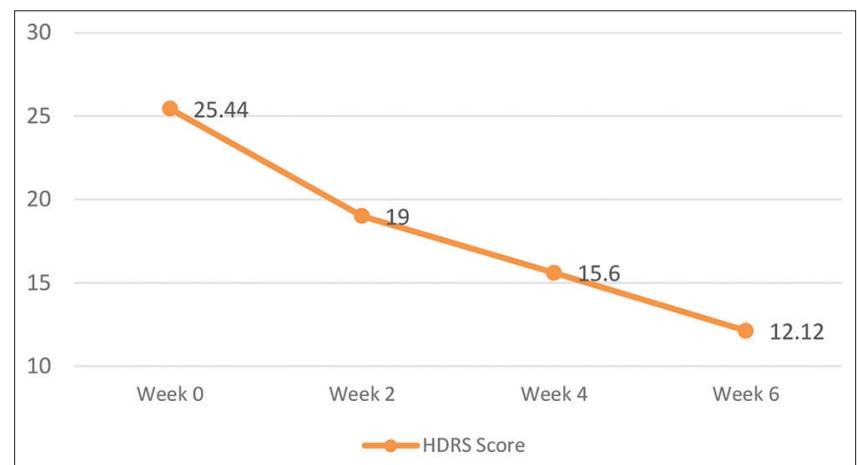

Figure 5: Comparison of Hamilton depression rating scale score week $0,2,4$, and 6
Patients with depression might have elevation in SAA level [13], [14]

After 2 weeks administration of fluoxetine, the measurement showed a significant reduction of sAA levels. The measurement in the $4^{\text {th }}$ week and the $6^{\text {th }}$ week also showed a significant reduction $(p<0.001)$. Decrease of the enzyme levels of sAA was noticeable after fluoxetine administration. The minimum levels of sAA enzymes in depressed subjects before therapy were $22 \mathrm{kU} / \mathrm{L}$ and the maximum was $99 \mathrm{kU} / \mathrm{L}$, indicating a considerable wide range of value. The difference in the levels of sAA enzymes among the subjects could be due to the sampling of sAA (09.00 Until 14.00) so that various factors could affect the level of the sAA enzyme, including the waiting time, hunger conditions, eating or drinking products that could increase the concentration of sAA, smoking, etc. [10]. This could lead to a bias of the results and become the limitation on this study.

Our data also showed that salivary cortisol levels in depressed subjects were higher compared to healthy controls. Our finding was consistent with those from Ishitobi et al. (2010) which found that sAA and cortisol levels in unremitted patients were significantly elevated compared to controls and remitted patients [13]. The mean value of salivary cortisol showed a reduction in the $4^{\text {th }}$ week and the $6^{\text {th }}$ week of measurements. So that it could be concluded that there was a significant reduction of salivary cortisol with $p<0.001$ after fluoxetine administration. The molecular mechanisms underlying the SSRI function were unknown. However, SSRIs can affect the endocrine and immune system of patients with depression. In vitro studies had shown that SSRIs enhanced transcription of the mediated GR in the presence of cortisol and proposed that the antidepressant inhibits the transporter of steroid-bound membranes, increasing the intracellular concentration of glucocorticoids, which in turn increase the expression and function of GR and restore negative feedback by cortisol [22], [23].

Similarly, HDRS scores were significantly decreased in the $2^{\text {nd }}$, the $4^{\text {th }}$, and the $6^{\text {th }}$ week with $p<0.001$. The effectiveness of fluoxetine administration was confirmed by the reduction of HDRS score compared to initial values. There was a $52.36 \%$ decline of initial HDRS score after 6 treatment. This is in line with the study by Piwowarska et al., which showed reduction in the HDRS score which could be seen starting from the $2^{\text {nd }}$ week. The patient who successfully treated with fluoxetine showed a decline of $50 \%$ HDRS score and decreased secretion of cortisol in the $6^{\text {th }}$ and $8^{\text {th }}$ weeks [19].

This study tried to minimize factors that could affect the levels of the sAA enzyme and salivary cortisol, including chronic metabolic diseases, oral diseases, the use of drug agonists or adrenergic antagonists, corticosteroid therapies, and comorbidities with other psychiatric disorders. There were still many things that could cause bias on this study that has been mentioned previously. 
This study used saliva cortisol with consideration that it is easy, inexpensive; the subject can take its own sample. The blood serum is not carried out with the reason of additional stress, which can affect the measurement of sAA level which is also done in the same time. We used passive drooling technique into sterile pots. This method is able to provide samples in large quantities and is easy to do, but the weaknesses are containing mucin and caused an unpleasant smell. The average study uses a saliva retrieval method with an absorbent material such as Salivette ${ }^{\circledR}$ but it is not currently available in Indonesia.

This study should be replicated using another group of antidepressants because it is cheap and easy to compare to another method. In the future, objective examination of sAAenzyme levels was expected to be an additional routine that could help determine depression level and monitoring the progress of treatment.

\section{Conclusions}

Our findings support previous studies that the sAA levels and the salivary cortisol levels were higher in depression compared to healthy controls. The sAA and the cortisol level were significantly decreased after fluoxetine administration followed by at least $50 \%$ reduction of HDRS scores after 6 weeks of fluoxetine administration. Measurement of sAA levels could be used as a potential biomarker of therapeutic response in depressed patients in addition to salivary cortisol.

\section{Authors' Contributions}

All the authors were involved in the conception of the study. AJT, HM, and MS were involved in interpretation of the research findings. SS and STL were contributed to the drafting of the manuscript. All authors read and approved the final manuscript.

\section{Acknowledgments}

The author would like to thanks participants who volunteered for this study and participated in the data collection. The authors would like to acknowledge the important support and contributions of Arifin Seweng.

\section{Ethics Approval and Consent to Participate}

The research has been permitted and acknowledged by Hasanuddin University Ethic Medical Committee. Before each interview, each participant was given written information on the study. Each participant was also informed that his or her participation was voluntary. Before each interview, we emphasized the importance of maintaining confidentiality in relation to patient cases. All participants provided written consent to participate in the study.

\section{References}

1. World Health Organization. Depression. Geneva: World Health Organization; 2018. Available from. https://www.who.int/newsroom/fact-sheets/detail/depression. [Last accessed on 2018 Dec 10].

2. Indonesian Ministry of Health. Basic Health Research Indonesia: Indonesian Ministry of Health; 2018. Available from: $\quad$ http://www.depkes.go.id/resources/download/infoterkini/materi_rakorpop_2018/Hasil\%20Riskesdas \%20 2018.pdf. [Last accessed on 2018 Dec 10]. https://doi. org/10.17501/24246735.2018.4105

3. PutraST.Biologyperception.In:MedicalPsychoneuroimmunology $2^{\text {nd }}$ ed. Surabaya: Airlangga University Press; 2011. p. 33-41.

4. Won E, Kim Y. Stress, the autonomic nervous system, and the immune-kynurenine pathway in the etiology of depression. Curr Neuropharmacol. 2016;14(7):665-73. https://doi.org/10.2174/15 70159x14666151208113006 PMid:27640517

5. Ali N, Pruessner JC. The salivary alpha amylase over cortiso ratio as marker to assess dysregulations of the stress system. Physiol Behav. 2012;106(1):65-72. https://doi.org/10.1016/j. physbeh.2011.10.003

PMid:22019784

6. Sharpley CF. Neurobiological pathways between chronic stress and depression: Dysregulated adaptive mechanisms? Clin Med. 2009;2:33-45.

7. Fried EI, Nesse RM. Depression sum-scores don't add up: Why analyzing specific depression symptoms is essential. BMC Med. 2015;13(1):72. https://doi.org/10.1186/s12916-015-0325-4

8. Hacimusalar Y, Eşel E. Suggested biomarker for major depressive disorder. Arch Neuropsychiatry. 2018;55(3):280-90. PMid:30224877

9. Chrousos GP. Stress and disorders of the stress system. Nat Rev Endocrinol. 2009;5(7):374-81. PMid:19488073

10. Booij SH, Bos EH, Bouwmans ME, Van Faassen $M$, Carr Kema LP, Oldehinkel AJ, et al. Cortisol and $\alpha$-amylase secretion patterns between and within depressed and nondepressed individual. PLoS One. 2015;10(7):e0131002. https:// doi.org/10.1371/journal.pone.0131002 PMid:26148294

11. Stetler C, Miller GE. Depression and hypothalamic-pituitaryadrenal activation: A quantitative summary of four decades of research. Psychosom Med. 2011;73(2):114-26. https://doi. 
org/10.1097/psy.0b013e31820ad12b

PMid:21257974

12. Schumacher S, Kirschbaum C, Fydrich T, Ströhle A. Is salivary alpha-amylase an indicator of autonomic nervous system dysregulations in mental disorders? A review of preliminary findings and the interactions with cortisol. Psychoneuroendocrinology. 2013;38(6):729-43. https://doi. org/10.1016/j.psyneuen.2013.02.003 PMid:23481259

13. Ishitobi Y, Akiyoshi J, Tanaka Y, Ando T, Okamoto S, Kanehisa M, et al. Elevated salivary $\alpha$-amylase and cortisol levels in unremitted and remitted depressed patients. Int J Psychiatry Clin Pract. 2010;14(4):268-73. https://doi.org/10.3109/1365150 1.2010 .500737

PMid:24917438

14. Bauduin SE, van Noorden MS, van der Werff SJ, de Leeuw M, van Hemert AM, van der W NJ, et al. Elevated salivary alpha amylase level at awakening in patient with depression. Psychoneuroendocrinology. 2018;97:69-77. https://doi. org/10.1016/j.psyneuen.2018.07.001 PMid:30005283

15. Vermetten $E$, Vythilingam $M$, Schmahl $C D$, Southwick $S M$, Charney DS, Bremner JD. Alterations in stress reactivity after long-term treatment with paroxetine in women with posttraumatic stress disorder. Ann NY Acad Sci. 2006;1071:184-202. https://doi.org/10.1016/s0006-3223(03)00634-6

PMid: 16891570

16. Ruhe HG, Khoenkhoen SJ, Ottenhof KW, Koeter MW, Mocking RJ, Schene AH. Longitudinal effects of SSRI paroxetine on salivary cortisol in major depressive disorder. Psychoneuroendocrinology. 2015;52:261-71. https://doi. org/10.1016/j.psyneuen.2014.10.024

PMid:25544738

17. Deuschle M, Hamann B, Meichel C, Krumm B, Lederbogen F, Kniest $A$, et al. Antidepressive treatment with amitriptyline and paroxetine: effects on saliva cortisol concen-trations. J Clin Psychopharmacol. 2003;23(2):201-5. https://doi. org/10.1097/00004714-200304000-00014

PMid:12640223

18. Appelhof BC, Huyser J, Verweij M, Brouwer JP, Van Dyck R, Fliers E, et al. Glucocorticoids and relapse of major depression (dexamethasone/corticotropin-releasing hormone testin relation to relapse of major depression). Biol Psychiatry. 2009;59(8):696-701. https://doi.org/10.1016/j.biopsych.2005.09.008

PMid:16368077

19. Piwowarska J, Chimiak A, Matsumoto H, Dziklińska A, Radziwoń-Zaleska M, Szelenberger W, et al. Saliva cortisol concentration in patient with major depression after treatment with fluoxetine. Psychiatr Res. 2012;198(3):407-11. https://doi. org/10.1016/j.psychres.2012.01.029

PMid:22429479

20. Veen G, Giltay EJ, Licht CM, Vreeburg SA, Cobbaert CM, Penninx BW, et al. Evening salivary alpha-amylase, major depressive disorder, and antidepressant use in the Netherlands study of depression and anxiety (NESDA). Psychiatr Res. 2013;208(1):41-6. https://doi.org/10.1016/j. psychres.2013.03.012

PMid:23587658

21. Lisal ST, Azis U, Thioritz W, Idrus MF, Tanra AJ. The comparison of salivary alpha amylase enzym level between anxiety patients and depression patients. Int J Sci. 2017;36:334-44.

22. Hernández ME, Mendieta D, Martínez-Fong D, Loría F, Moreno J, Estrada I, et al. Variations in circulating cytokine levels during 52 week course of treatment with SSRI for major depressive disorder. Eur Neuropsychopharmacol. 2008;18(12):917-24. https://doi.org/10.1016/j.euroneuro.2008.08.001

PMid:18805677

23. Blardi P, De Lalla A, Leo A, Auteri A, lapichino S, Di Muro A, et al. Serotonin and fluoxetine levels in plasma and platelets after fluoxetine treatment in depressive patients. J Clin Psychopharmacol. 2002;22(2):131-6. https://doi. org/10.1097/00004714-200204000-00005

PMid:11910257 